\title{
Symmetric Versus Nonsymmetric Structure of the Phosphorus Vacancy on InP(110)
}

\author{
Ph. Ebert, ${ }^{1}$ K. Urban, ${ }^{1}$ L. Aballe, ${ }^{2}$ C. H. Chen, ${ }^{2}$ K. Horn, ${ }^{2}$ G. Schwarz, ${ }^{2}$ J. Neugebauer, ${ }^{2}$ and M. Scheffler ${ }^{2}$ \\ ${ }^{1}$ Institut für Festkörperforschung, Forschungszentrum Jülich GmbH, D-52425 Jülich, Germany \\ ${ }^{2}$ Fritz-Haber-Institut der Max-Planck-Gesellschaft, Faradayweg 4-6, D-14195 Berlin-Dahlem, Germany
}

(Received 30 November 1999)

\begin{abstract}
The atomic and electronic structure of positively charged $\mathrm{P}$ vacancies on $\mathrm{InP}(110)$ surfaces is determined by combining scanning tunneling microscopy, photoelectron spectroscopy, and density-functional theory calculations. The vacancy exhibits a nonsymmetric rebonded atomic configuration with a charge transfer level $0.75 \pm 0.1 \mathrm{eV}$ above the valence band maximum. The scanning tunneling microscopy (STM) images show only a time average of two degenerate geometries, due to a thermal flip motion between the mirror configurations. This leads to an apparently symmetric STM image, although the ground state atomic structure is nonsymmetric.
\end{abstract}

PACS numbers: 73.20.Hb, 61.16.Ch, 71.15.Mb, 79.60.-i

Although it is well known that point defects can exert a profound influence on the physical properties of semiconductors, the determination of the atomic scale geometric and electronic structure of point defects has remained a challenging task for theoretical as well as experimental research. One particularly striking example is anion vacancies on (110) surfaces of III-V semiconductors, where no agreement has been reached regarding even basic properties, such as (i) the symmetry of the atomic structure and (ii) the energy of defect levels in the band gap: Although recent density-functional theory (DFT) calculations for positively charged arsenic vacancies on the GaAs(110) surface agreed that the gallium atoms neighboring the vacancy relax into the surface layer (in contrast with an earlier tight-binding calculation [1]), one calculation found a rebonded configuration breaking the mirror symmetry of the surface [2], whereas the other predicted a fully symmetric configuration to have the lowest energy [3]. High resolution scanning tunneling microscopy (STM) images show a density of states preserving the mirror symmetry of the surface at the defect site $[1,4]$. Although this seems to favor a symmetric atomic structure, the experimental results could not be matched to results of any of the DFT calculations [5-8]. Furthermore, scanning tunneling spectroscopy (STS) yielded a local downward band bending of $0.1 \mathrm{eV}$ [1], whereas surface photovoltage measurements found a band bending of $0.53 \pm 0.03 \mathrm{eV}$ [9] at the site of the positively charged As vacancy on $p$-doped GaAs(110) surfaces. Concerning the energy levels, the two different DFT calculations predicted the charge transfer levels $(+/ 0)$ to be $0.32 \mathrm{eV}$ [2] and $0.1 \mathrm{eV}$ [3], and the lowest Kohn-Sham eigenvalues in the band gap to be $0.73 \mathrm{eV}$ [2] and $0.06 \mathrm{eV}$ [3] above the valence band maximum (VBM). In view of this puzzling situation it is obvious that the interpretation of the experimental STM images as well as the structure of the anion vacancies on (110) surfaces of III-V semiconductors is still under debate [5-8].

Discrepancies such as those pointed out above can arise due to limitations of the methods used. On the theoretical side DFT calculations have proven to be powerful to deter- mine the structure of point defects [10]. Yet differences in the size of the supercell, the pseudopotentials employed, or the exchange-correlation functional implemented might lead to different results, such as the defect's symmetry or the position of the defect levels, especially in situations where two configurations are almost degenerate in energy. On the experimental side the STM images alone do not provide direct information about the atomic structure nor are the experimental conditions, notably the concentration of the vacancies, the same in all experiments. Furthermore, the quantities discussed to describe the position of the defect level, i.e., Kohn-Sham eigenvalues within DFT, band bending, and charge transfer levels, are different physical quantities and thus (typically) deviate from each other significantly. In addition, the tip of the STM may affect the structure of the vacancy, since it is well known that the tip can even excite vacancies to migrate [11]. Therefore the combination of state-of-the-art theory and well-defined experiments is needed to get reliable results and test the correct interpretation of each of the separate results.

In this Letter we combine three different methods to determine the geometric and electronic structure of phosphorus $(\mathrm{P})$ vacancies on $p$-doped $\operatorname{InP}(110)$ surfaces: STM, photoelectron spectroscopy (PES), and DFT. This enables us to determine the charge transfer level of the defect state in the band gap and the equilibrium atomic structure of the vacancy in $p$-type material. We identify the rebonded structure as the ground state of the positively charged defect and demonstrate that a thermal flip motion between two mirror configurations results in simulated STM images consistent with the experimental findings. Our results solve the apparent discrepancies of the previous experimental and theoretical studies [12], provide a comprehensive picture of the vacancy structure, and point out a methodology to determine the structure of defects with high accuracy.

The surface vacancies were produced in high concentrations by annealing initially defect-free $p$-doped $\operatorname{InP}(110)$ cleavage surfaces [carrier concentration $n_{\mathrm{dop}}$ of (1.3$\left.2.1) \times 10^{18} \mathrm{~cm}^{-3}\right]$ at $160{ }^{\circ} \mathrm{C}[13,14]$. Figure 1 shows that 


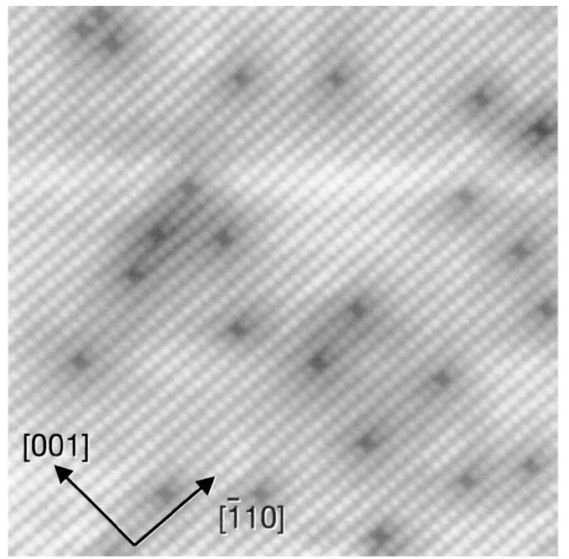

FIG. 1. Occupied state STM image of $\mathrm{P}$ monovacancies formed by annealing $p$-doped $\operatorname{InP}(110)$ surfaces at $160{ }^{\circ} \mathrm{C}$. The image has been acquired at $-2.7 \mathrm{~V}$ tunneling voltage and $0.9 \mathrm{nA}$ current.

this procedure leads indeed to only one type of defect on the surface which was identified as a singly positively charged phosphorus vacancy previously and whose concentration can be controlled by the annealing time [13-15]. We prepared several samples from the same wafer with a variety of annealing times in an ultrahigh vacuum (UHV) chamber at the "Berliner Elektronenspeicherring für Synchrotronstrahlung" storage ring for the photoelectron spectroscopy and in another UHV system for the STM measurements ensuring an exact control of defect concentration in both experiments. All measurements were done at room temperature. Angle-resolved PES measurements were used to probe the valence band in normal emission at a photon energy of $50 \mathrm{eV}$, and the indium $4 d$ core level. The InP sample was in Ohmic contact with a Au metal surface cleaned by scratching, on which the position of the Fermi level was measured. With the STM we measured constant-current images at a variety of voltages. From these images the vacancy concentrations were determined as a function of the annealing time [13] as well as the spatial distribution of the occupied and empty density of states of the vacancies with atomic resolution.

We first focus on the determination of the energy of the defect state. Here we concentrate on the charge transfer level of the surface vacancy, which is defined as the position of the surface Fermi level at which the defect changes its charge state. This energy level can be calculated by DFT and can be probed by measuring the band bending as shown below. We note that the energy of the charge transfer level might be very different from that of the ionization levels or the Kohn-Sham eigenvalues within DFT. In particular, large deviations between these quantities might occur in systems with large atomic relaxations such as surface and bulk defects [10].

STM images reveal that during heat treatment of initially defect-free cleavage surfaces the vacancy concentration increases with increasing annealing time. Similarly, the position of the Fermi level, which on well-cleaved defect-free surfaces is close to the top of the valence band (VBM) for $p$-type samples, is found to shift toward midgap upon annealing, as measured by PES (see Fig. 2). The filled diamonds (left axis) show the band bending at the surface, as determined through PES, whereas the open circles show the vacancy concentration (from STM, right axis) as a function of the annealing time. The band bending reaches a saturation value of $0.65 \mathrm{eV}$ at vacancy concentrations of $5 \times 10^{12} \mathrm{~cm}^{-2}$. Figure 2 also demonstrates that the Fermi level shift is directly correlated to the increase of the vacancy concentration.

By combining the vacancy concentration and the band bending it induces, it is possible to determine the energy of the charge transfer level in the band gap $E_{s d}$, because the charge per surface area in the surface layer $Q_{s s}$ is exactly compensated by the charge density per surface area in the depletion layer $Q_{s c}$ [16]. The charge per surface area induced by a concentration $n_{s d}$ of $+1 e$ charged $\mathrm{P}$ surface vacancies is [16]

$$
Q_{s s}=\frac{e n_{s d}}{\exp \left[\left(E_{F}-E_{s d}\right) / k T\right]+1},
$$

where $E_{F}$ is the Fermi energy. The difference in energy of the charge transfer level and the Fermi level $E_{s d}-E_{F}$ is given by $\left(E_{s d}-E_{s v}\right)-\left(E_{v}-E_{s v}\right)-\left(E_{F}-E_{v}\right)$ (for definitions, see inset in Fig. 2; $E_{v}$ and $E_{s v}$ are the positions of the valence band in the bulk and at the surface, respectively) where $E_{v}-E_{s v}$ is the band bending $e V_{s}$ measured by photoelectron spectroscopy at the surface. The charge density $Q_{s c}$ in the depletion layer compensating $Q_{s s}$ is

$$
Q_{s c}=\sqrt{2 \epsilon_{0} \epsilon_{r} n_{\mathrm{dop}} k T\left[\exp \left(\frac{-e V_{s}}{k T}\right)+\frac{e V_{s}}{k T}-1\right]} .
$$

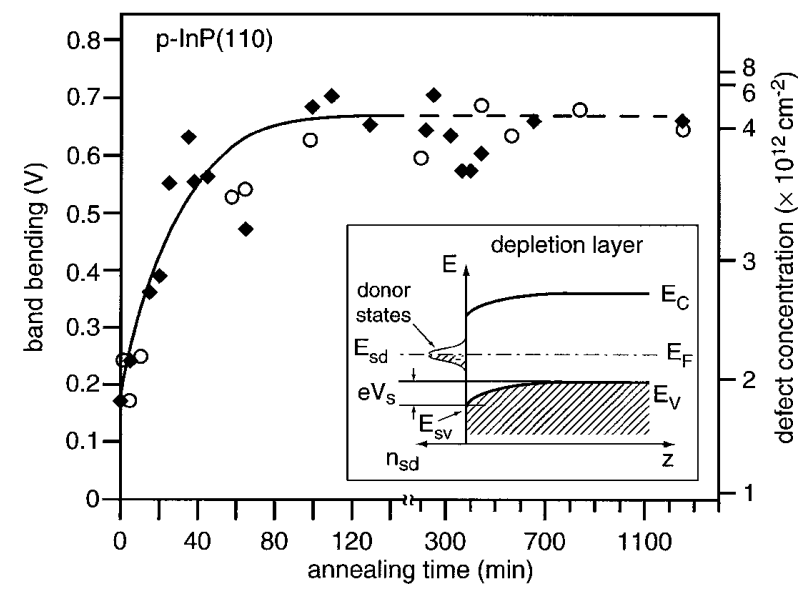

FIG. 2. The band bending (filled diamonds, left axis) and the vacancy concentration (empty circles [13], right axis) on the InP(110) surface as a function of the annealing time at $160{ }^{\circ} \mathrm{C}$. The solid line is the vacancy concentration calculated on the basis of Eqs. (1) and (2) and a fit through the measured band bending values. The dashed line shows the saturation value. The right scale is chosen such that according to Eqs. (1) and (2) the vacancy concentration is shown on a scale linear proportional to the band bending. The inset shows the schematic drawing of the depletion region in case of a fully pinned surface. 
The energy difference between the Fermi energy and the valence band maximum in the bulk has been determined for a nondegenerate InP crystal from the carrier concentration according to

$$
E_{F}-E_{v}=-k T \ln \left[4 n_{\mathrm{dop}}\left(\frac{2 m_{p} k T}{\pi \hbar^{2}}\right)^{-3 / 2}\right]
$$

with $m_{p}$ being the mass of the holes.

These equations permit a determination of the difference in energy of the charge transfer level $E_{s d}$ and the valence band maximum at the surface $E_{s v}$ using the vacancy concentration dependence of the band bending (see Fig. 2). We obtain an energy for the charge transfer level of the $\mathrm{P}$ vacancy of $0.75 \pm 0.1 \mathrm{eV}$ above the valence band maximum at room temperature. Note that the knowledge of the defect concentrations is crucial to determine the charge transfer level from a measurement of the band bending.

At this stage we compare this energy value with results from our total-energy density-functional theory calculations. Details of the calculational method used are described in Refs. [17,18]. Here we only increased the cutoff of the plane wave basis set to $15 \mathrm{Ry}$ in the electronic structure calculation to ensure convergence of the indium pseudopotential. We used a $2 \times 4$ and six layers thick supercell, which is large enough here, since the calculated bond lengths changed less than $0.01 \AA$ and the defect formation energy by less than $0.01 \mathrm{eV}$ when increasing the size of the supercell. For the $+1 e$ charged $P$ vacancy the calculation yields two different equilibrium atomic configurations, whose total energies differ by only $0.07 \mathrm{eV}$. The vacancy configuration with the lowest energy exhibits a rebonding of one of the neighboring surface atoms with the indium in the second layer leading to a nonsymmetric atomic structure with respect to the (110) mirror plane of the defect-free (110) surface [see the atomic structure superposed in Fig. 3(a)]. The calculated indiumindium spacing of this configuration is $2.89 \AA$ as compared to $2.98 \AA$ for the symmetric ground state structure of the neutral defect. A similar relaxation has also been calculated for the positively charged As vacancy on GaAs(110) $[2,12]$. From our calculations we find a charge transfer level $(+/ 0)$ at $0.52 \mathrm{eV}$ above the VBM for the nonsymmetric vacancy. For the energetically less stable symmetric configuration this level is at $0.45 \mathrm{eV}$. Both values deviate from the measured charge transfer level by roughly $0.2 \mathrm{eV}$. We attribute this deviation to the well-known underestimation of energy levels in local density approximation. An approximate upper limit of the error is the difference between the calculated and experimental band gap. Using our calculated value $(1.26 \mathrm{eV})$ and the experimental one $(1.42 \mathrm{eV})$ we get a systematic error of about $0.16 \mathrm{eV}$. We can therefore conclude that the theoretical and experimental positions of the defect levels agree well within the error margins. We note, however, that the systematic error for the calculated energies of the charge transfer levels is too large to identify the symmetry of the vacancy on the position of the defect level only. In the following we will show

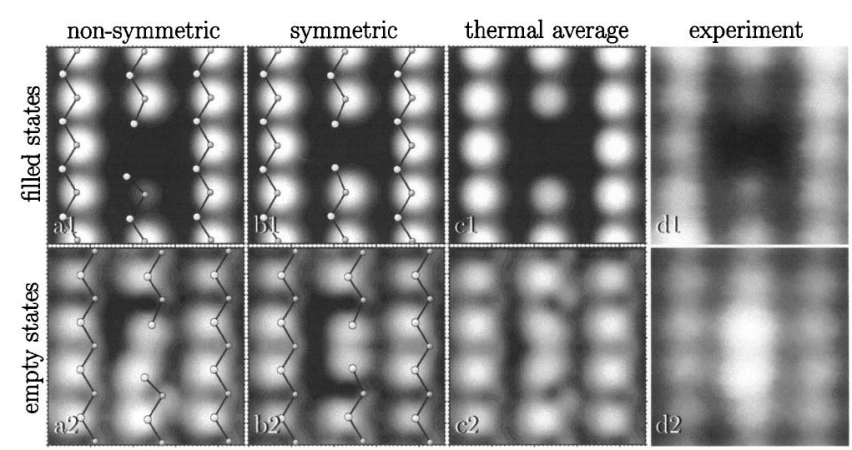

FIG. 3. Simulated STM images deduced from the calculated local density of states $3 \AA$ above the surface of a $+1 e$ charged $P$ vacancy on $\operatorname{InP}(110)$ surfaces for different atomic configurations (a) -(c) and experimental high resolution STM images of a $P$ vacancy measured at $(\mathrm{d} 1)-2.9$ and $(\mathrm{d} 2)+2.0 \mathrm{~V}$. The simulation shows the occupied (upper frames, energy range from VBM to $\mathrm{VBM}-1 \mathrm{eV}$ ) and empty (lower frames, energy range from VBM to VBM $+2 \mathrm{eV}$ ) states for (a1),(a2) a nonsymmetric rebonded; (b1),(b2) a symmetric nonrebonded; and (c1),(c2) the time average of the two mirror configuration of the nonsymmetric rebonded vacancy configuration. The respective atomic configurations are superposed on the simulated images.

that an unambiguous identification can be obtained based on a comparison of the total energies and an analysis of the STM micrographs.

We now focus on the atomic structure. STM images of $+1 e$ charged $\mathrm{P}$ vacancies [Fig. 3(d)] always exhibit an apparently symmetric density of states with respect to the (110) mirror plane. The STM images thus suggest a symmetric vacancy structure although in our calculations we find the nonsymmetric structure to be lower in energy. In order to clarify this apparent conflict, we have calculated in Figs. 3(a)-3(c) simulations of occupied (upper frames) and empty (lower frames) state STM images from the local density of states [17] of different vacancy configurations. Frames 3(a1) and 3(a2) show the simulated STM images for the nonsymmetric case, whereas frames 3(b1) and 3(b2) show the same for the symmetric vacancy configuration. Both vacancy configurations lead to clearly distinguishable STM images: The nonsymmetric vacancy exhibits a pronounced asymmetry. The $\mathrm{P}$ atom next to the rebonded indium atom [upper atom in Fig. 3(a)] has a brighter dangling bond than the $\mathrm{P}$ atom at the opposite side of the vacancy and the rebonded indium atom has a weaker maximum in the empty states. Lateral displacements are also visible. These effects are purely electronic, because the calculated height difference is only 0.11 and $0.07 \AA$ for the cations and anions, respectively. In contrast, the symmetric vacancy shows the expected symmetry with respect to the (110) mirror plane [Fig. 3(b)]. The images of the occupied states exhibit very little change of the neighboring occupied dangling bonds [Fig. 3(b1)], and the separation of the two neighboring empty dangling bonds is considerably reduced [Fig. 3(b2)]. If we compare these simulations with the experimental STM images we find a symmetric density of states [Fig. 3(d)] in conflict with the 
energetically favorable nonsymmetric vacancy configuration in Fig. 3(a). However, also the symmetric vacancy configuration does not agree with the experimental STM images: The empty state STM images do not show any reduced separation of the two neighboring empty dangling bonds in contrast to the simulation of the symmetric vacancy structure [Fig. 3(b2)]. Thus none of the static equilibrium vacancy configurations can be matched in detail to the experimental observation.

This conflict can be resolved by closer inspection of the barrier between the two possible mirror configurations of the asymmetric vacancy. We estimated an upper limit for this barrier by mapping the energy along the direct reaction path between the symmetric and nonsymmetric vacancy configurations. On this basis we found an upper limit of $0.08 \mathrm{eV}$ for the barrier between the two nonsymmetric mirror configurations. The low value of the barrier implies that, at room temperature, the vacancy flips between the two nonsymmetric configurations at a rate significantly higher than the time resolution of the STM (1 to $10 \mathrm{~Hz})$. We estimate a flip rate at room temperature of $0.1 \mathrm{THz}$ using the transition state theory, the optical phonon frequency of $2 \mathrm{THz}$ [19] as attempt frequency, and the calculated barrier. This concept of a thermally activated flip motion has been well established for dimers at the $\mathrm{Si}(001)$ surface [20]. Based on these findings we interpret the STM image as a time average of the flipping vacancy.

In order to test the model of a thermal flip motion we have performed STM image simulations based on this flip mechanism. In Fig. 3(c) we assumed that the vacancy flips such that the density of states probed by the STM is the average of the rebonded and nonrebonded sides of the vacancy (average of the two mirror configurations). The images agree very well with the experiment. In particular, two major features - the nearly unchanged separation of the neighboring empty dangling bonds [Fig. 3(c2)] and the depression of the neighboring occupied dangling bonds [Fig. 3(c1)] - are well reproduced. Close inspection of Figs. 3(c) and 3(d) clearly demonstrates that the images of the thermally flipping vacancy configuration explains the STM images best. Note that the somewhat higher intensity in the experimental empty state images is due to the local electrostatic potential arising from the positive charge [2] and that the small intensity of empty density of states above the anions decays quickly into the vacuum such that the STM cannot probe it [21].

In conclusion, we have demonstrated a methodology to determine the atomic structure and the energy of charge transfer levels in the band gap of surface defects, by combining scanning tunneling microscopy, photoelectron spectroscopy, and density-functional theory calculations. From linking STM and PES data we find a charge transfer level $(+/ 0) 0.75 \pm 0.1 \mathrm{eV}$ above the VBM for the example of $P$ vacancies on $\operatorname{InP}(110)$ in good agreement with DFT calculations. By comparing high resolution STM images with calculations, we determine the vacancy to exhibit a rebonded nonsymmetric structure. The vacancy flips ther- mally between its two mirror configurations. This localized vibrational mode leads to an apparently symmetric density of states in STM images, although the vacancy has a nonsymmetric atomic structure. Similar measurements may help one to understand on the atomic scale the properties of a wide range of materials, where defects play a key role.

We thank Th. Chassé for assistance in an initial experiment, W. Mönch for valuable discussions, and K. H. Graf for technical support. This work was supported by the Bundesministerium für Forschung und Technologie under Grant No. 05SE8 OLA7 and by the Deutsche Forschungsgemeinschaft through Sonderforschungsbereich 296 TP A4 and A5 and Grant No. Ne428/2-1.

[1] G. Lengel et al., Phys. Rev. Lett. 72, 836 (1994).

[2] S. B. Zhang and A. Zunger, Phys. Rev. Lett. 77, 119 (1996).

[3] H. Kim and J. R. Chelikowsky, Phys. Rev. Lett. 77, 1063 (1996); Surf. Sci. 409, 435 (1998).

[4] Ph. Ebert, K. Urban, and M. G. Lagally, Phys. Rev. Lett. 72, 840 (1994).

[5] J. Harper, G. Lengel, R. E. Allen, and M. Weimer, Phys. Rev. Lett. 79, 3312 (1997).

[6] S. B. Zhang and A. Zunger, Phys. Rev. Lett. 79, 3313 (1997).

[7] J. Harper, G. Lengel, R. E. Allen, and M. Weimer, Phys. Rev. Lett. 79, 3314 (1997).

[8] H. Kim and J. R. Chelikowsky, Phys. Rev. Lett. 79, 3315 (1997).

[9] S. Aloni, I. Nevo, and G. Haase, Phys. Rev. B 60, R2165 (1999).

[10] U. Scherz and M. Scheffler, in Imperfections in III-V Materials, edited by E. R. Weber (Academic Press, New York, 1993).

[11] Ph. Ebert, Surf. Sci. Rep. 33, 121 (1999).

[12] The theoretical results for the positively charged As vacancy on GaAs(110) exhibit a very close agreement to those for the respective $\mathrm{P}$ vacancy on $\operatorname{InP}(110)$. Thus both vacancies can be expected to show a thermal flip motion resulting in good agreement between STM simulation and experiment.

[13] Ph. Ebert et al., Phys. Rev. B 51, 9696 (1995).

[14] Ph. Ebert et al., Phys. Rev. B 53, 4580 (1996).

[15] Ph. Ebert et al., Phys. Rev. Lett. 76, 2089 (1996).

[16] W. Mönch, Semiconductor Surfaces and Interfaces, Springer Series in Surface Sciences Vol. 26, edited by G. Ertl et al. (Springer-Verlag, Berlin/Heidelberg, 1993).

[17] G. Schwarz, A. Kley, J. Neugebauer, and M. Scheffler, Phys. Rev. B 58, 1392 (1998).

[18] M. Bockstedte, A. Kley, J. Neugebauer, and M. Scheffler, Comput. Phys. Commun. 107, 187 (1997); see also http:// www.fhi-berlin.mpg.de/th/fhimd/

[19] U. Schröeder, J. Fritsch, and P. Pavone, Physica (Amsterdam) 219B-220B, 434 (1996); H. Nienhaus and W. Mönch., Surf. Sci. 328, L561 (1995).

[20] J. Dąbrowski and M. Scheffler, Appl. Surf. Sci. 56-58, 15 (1992).

[21] B. Engels et al., Phys. Rev. B 58, 7799 (1998). 